trust, we strive to acquit ourselves, at least, in such a manner as shall not disgrace the college and the trust.

There is only one Fellow of the College to whom I need refer in the obituary which is associated with this lecture, but I should be very sorry to omit all mention of his name. Christopher Heath was a very active and useful member of the Council of the College for many years, and became its President in 1895. In 1897 he delivered the Hunterian Oration. His books on Practical Anatomy and Minor Surgery early gained for him a reputation as a teacher, and he retained that reputation throughout his career at University College Hospital. Indetd, he added to it by the directness of his speech and the clearness of his views. I believe he would have attained greater success as a surgeon had he belonged wholly to the preantiseptic or the post-antiseptic period. But, coming as he did between the two, he found it difficult to adapt himself to the altered conditions which the vast change of thought and practice imposed on our profession. Even as it was he was justly regarded as a leader of surgery in this town, and as one of the boldest operators of his day. Those of us who knew him well miss his fine presence and his cheery manner, while the vigour of his speech was always pleasing to us, whether he was on our side or in opposition to our views.

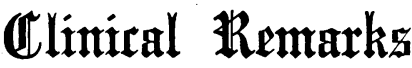 \\ os}

\section{ICHTHYOSIS AND ITS TREATMENT.}

BY W. ALLAN JAMIESON, M.D., F.R.C.P.E.,

CONSULTING PHYSICIAN FOR DISEASES OF THE SKIN, EDINBURGH ROYAL INFIRMARY.

VARIATIONS in the condition of the human integument as regards unctuosity and dryness are considerable, though in the civilized races, at least, such are not, under normal circumstances, very noticeable. In those persons who bathe regularly, the skin of the trunk does not exhibit any pronounced greasiness such as would manifestly soil tissue paper. The lubricity of the skin is greatest in adolescence and early manhood, diminishing with age. Undoubtedly, however, both inherited qualities and habits of life exert an influence on its proportion. Individuals with thick, coarse skins have likewise the oiliest integument, and alcoholic indulgence, if excessive, and particularly in those who lean to the obese type, is apt to augment this where the tendency already exists, probably in part by so constantly maintaining vascular tension in the peripheral vessels at a low ebb.

Extreme dryness of the skin is less frequent. It is met with in the old, as a portion of that atrophy of the cutaneous envelope with lessened glandular activity which is the heritage of advancing years, while it is sometimes encountered in persons endowed with a very thin cataneous investment, in whom the physiological separation of the effete keratinous particles is not correspondingly replaced by sufficient growth from beneath. Such stand cold weather badly, their face and hands chap readily, and exposure to currents of air in motion, whether to biting winds, or in rapid transit as in motoring, is followed by pain and smarting in the exposed parts and occasionally even in regions better protected. Should they use much soap, especially the more ordinary forms of toilet soap, aggravation ensues, by too rapidly and unduly still further attenuating the delicate horny film.

We meet, however, in practice with individuals in whom the aridity of the skin reaches a point verging on, or actually in a state of, disease, and to the general aspect assumed in specially well-marked cases the term ichthyosis has been affixed. The commencement of this abnormality is laid in intranterine life, but is usually in abeyance till some months after birth, when it declares itself if uninterfered with, with ever-growing aggressiveness. There is an innate variety, the so-called ichthyosis congenita, admittedly rare; no instance has come directly under my observation. The following example is, however, hardly distinguishable from the intrinsic type.

J. H., 8 months, was sent to me at the Royal Infirmary on May 18th, 1905, by Dr. James Wilson. There was no history of any similar condition in any known relative. A brother somewhat older has a pecu'iarly fine and unusually trans- parent skin. When born the vernix caseosa was said to bave been particularly thick, so that the coating could not be completely washed off. 'The skin, however, though rougb, was thickened at birth. The scalp, when examined, was studded over with partly discrete, partly confluent, large vesicopustules. Tnese cccupy the top but not the sides of the head. The rest of the skin resembles in appearance nothing so closely as a peach, but to the finger it is rough, harsh, and filelike, not velvety and soft. It looks and feels oedematous, and when pinched up produces a thick fold between the fingers. It cannot be moved on the subcutaneous tissue, but gives the impression of a tough, doughy mass. The epidermis of the paims and soles is much thickened, coarse, and dry. There are deep furrows traceable in the skin between the plush-like structure. The child has cut his lower incisors, and the two upper central ones are nearly through the gum. He has been nursed by his mother. Has a tendency to diarrhoea. When the forehead is coated with oil, beads of perspiration can be seen on the layer of oil. The mother was directed to apply twice a day a mixture of resorcin, half a drachm in an ounce of glycerine of starch diluted with an equal quantity of glycerine.

He was brought back on July 6th. The treatment recommended caused slight improvement, which has not been main tained. The scalp, it is true, is now free from scabs and crusts, but the skin in general is rougher and more plush-like, while on the legs and also elsewhere here and there it is still further covered with a yellowish investment resembling seborrhoea. The child screams much, and is peevish and restless. He has cut two upper incisor teeth. Washing daily with resorcin salicylic soap was recommended previous to inunction. On October 5th, as the resorcin glycerine of starch did not suit, vaseline was substituted, the soap being persevere'd with. A considerable improvement was observable. On the head and face there were fewer crusts, though some yet exist on the chin and crown. The skin of the trunk and upper arms is thinner and more pliable. Even the forearms and legs are not so hard, and the skin is somewhat more movable, while still rough and plush-like. $\mathrm{He}$ is thinner and his appetite is capricious. Careful directions as to diet were given, which was to consist in the main of milk cooked with various farinaceous substances. He was not again seen, and died on May 15th, 1906. Dr. Wilson, in a letter received from him, says :

"The child, to my mind, seemed to die of a toxic condition, which I could only put down to the state of its epidermis. The skin over the arms and legs was very much thickened by layer upon layer of decomposing scales. The face was not quite so bad, and the rest of the body less so. The child had very rapid respirations for a considerable time, began to waste rapidly, became somewhat comatose, and died. The kidneys were all right; but it had a slight bronchial catarrh, which, however, did not account for the rapid respiration and wasting -hence my conclusion."

This seems, indeed, the most reasonable explanation.

In this case the hyperkeratosis was peculiarly well pronounced and developed at an earlier age than is customary, but there were also inflammatory phenomena associated with the excess of epidermic production, as shown by the vesico-pustules on the head when first seen, and by the decomposing crusts which accumulated towards the end of its life. According to Unna and Tommasoli, there are traces of inflammation in the upper layers of the corium in all cases of ichthyosis; thus there is nothing surprising that the evidences of this morbid condition should bave been present here. Thibierge ${ }^{1}$ observes that: "The general development of those affected with ichthyosis is subject to no universal rule. If a certain number, as several authors have remarked, are small and sickly, with poorly-developed muscles and bones; if sometimes the genital organs remain infantile throughout life, there are others who reach and exceed the medium height whose locomotor apparatus is remarkably vigorous, who exhibit no other anomaly save of the skin, and that even in the most accentuated forms of the disease." While this is in the main true, it seems to me that heredity, regimen, and environment modify this view materially. The dry, inelastic skin, not always growing commensurately with the internal parts, must militate against the attainment of a thoroughly robust frame. The following example illustrates this among other circumstances.

J. T., aged 16. Railway clerk in the country. Admitted to Royal Infirmary, October 17th, 1903. With exception of two younger hrothers, no near relative has any similar complaint. One brother is slightly. the other severely, affected. One brother and sister healthy. He has had measles, but is fairly robust.

Though not born with the disease, he has been subject to it as long as he can remember. The condition was apparently first noticed on his recovery from scarlet fever, when it was observed that he did not desquamate, but the skin grew dry, scaly, and rough on the surface. Whenever he takes a sharp walk, or does anything to make himself warm and give rise to 
sweating, he feels his whole body very itchy. Sometimes when he takes a bath the skin itches, at other times smarts. $\mathrm{He}$ is unable to say where he perspires when heated. He has never been able for very much exercise, since be becomes readily tired. Although tall his development is poor. He is narrow and markedly flat-chested. The muscles everywhere are flabby and soft. hands, and the plantar aspect of the feet, almost the whole surface is involved. While in many parts the condition does not go further than the presence of dry, dirty white or yellow epidermic plates, in certain situations the epidermic hypertrophy assumes the appearance of olive-green, rough, conical or blunt aggregations, which are very firmly attached. Thus, this is the character of the surface of the thighs on their inner side, on the knees, the popliteal spaces, the chest, abdomen, and back over the greater part, the legs, and dorsum of the feet. There is especial thickening on the outen aspects of the anterior and posterior axillary folds. The neck in front is little, but behind is considerably, involved. The scalp is thickly covered with dry flakes and scales, the hair is thin and short. The complexion is ruddy and the face quite plump and full. The temperature ranges between $96.8^{\circ}$ and $98.4^{\circ}$. The urine is only fairly copious, is acid, 1015, and contains no abnormal constituents.

The treatment ordered was washing, with friction, once daily, with a 5 per cent. resorcin and salicylic superfatted soap, and the inunction twice daily of dilute glycerine of starch, containing 1 drachm of resorcin in each ounce.

He was discharged home on November 7 th with his skin quite soft and smooth. On close inspection it was seen to be marked by very fine, hair-like lines, which enclosed whiter spaces. The lines were atrophic, caused by motion or friction between adjoining ichthyotic plates. The scalp was free from any scales or crusts, but the hair was still scanty. On the any scales or crusts, but the hair was still scanty. On the He was directed to wash several times a week with the soap, and to apply daily some of the resorcin cream with a view to obviate recurrence. In reply to a letter addressed to him inquiring as to his state, the following answer was received, dated January 1st, 1907 :

"I continued the treatment for some time after I came home, but when $T$. went to work in the factory I bad not the same chance, and the roughness came partly on again. About six months ago I went to work in a meal mill. It was hot weather at the time, and the perspiration brought it all off again. Since then I have got stronger and stouter, and am now able to carry a sack of meal without much trouble., My skin is as smooth as can be, and the hair has grown on it."

In this instance treatment was commenced before growth was completed, while the change which he made from a sedentary and indoor life to a more active and open-air one, combined with the start in the right direction which the skin had got during his residence in the Royal Infirmary, and the attention he gave it afterwards, made all the difference. What can be accomplished if the diseased state is tackled early enough, and correct management persisted in intelligently for a sufficient time, is shown in the next example, which, through the kindness of the family medical attendant, I have had the opportunity of seeing at intervals during ten years.

G. C., 2 years, first seen April, 1897. Has a sister and brother older, both with normal skins. She was brought up on the bottle, and to appearance is a rosy and healthy girl. Till she was a year old there was nothing unusual in the state of her skin. Her face then was noticed to be rough; shortly afterwards her legs became so also, and now the diseased condition has extended to the arms, shoulders, back, and chest. The skin over most of these parts is dry and file-like. The palms and soles present a natural aspect. The hair is plentiful, dark, and curly, but the scalp shows a considerable amount of dry dandruff. It is noticed that she never perspires. To commence with, she was washed each second night with resorcin and salicylic sosp, and anointed nightly with a salve composed of resorcin half a drachm, lanoline an ounce and a half, and almond oil half an ounce. The head was washed at intervals with yolk of egg and warm water, and almond oil with oil of eucalyptus applied.

In January, 1898, there was some improvement and the skin was smoother, but this was not maintained, since as soon as the treatment was intermitted the skin became again rough and dry, and the legs showed a tendency to hack. The scalp remained scaly. She was then, and has been at times since, liable to feverish attacks, the precise cause of which bas never been quite satisfactorily made out. During these, treatment for the skin had to be discontinued, and when convalescence was established the ichthyosis had recurred. The scalp was now directed to be washed frequently with an infusion of quillaia bark in warm water, and a pomade of sulphur and salicylic acid rubbed daily into the roots of the hair, while for the body the same combination of resorcin and glycerine 0 starch as was employed for the previously related cases was substituted for the salve. In February of the same year the skin was beautifully soft and smooth everywhere, and no trace of disease perceptible. The antumn was spent at the seaside, and it was observed that she perspired freely on warm days, beads of sweat standing on the surface or clinging to the lanugo hairs which were now growing plentifully, more particularly on the legs. Progress since then has been uninterrupted, till now, December, 1906, she is a remarkably wellgrown and strong girl of 12 , with epecially well-developed muscles. The legs are firm, straight and vigorous. The feverish attacks seem to have wholly cessed. Her skin is watched carefully, but needs little more than normal attention, only on cold frosty days does a slight sign of dryness show itself. There is no trace of scurf on the scalp, and the hair is kept in good condition by washing with the quillais infusion once in ten days, no oil or pomade being required.

In the following instance the rapidity of improvement under favourable circumstances was remarkable.

J. M., 4 years. Admitted to my ward on December 5th, 1905, at the recommendation of Dr. Calvert, Melrose. Mother states. that his skin was soft and smooth till be was 1 year old. Of children's complains he has only had whooping-cough. The disease started on the neck; which presented the appearance of not having been washed clean. There is no member of the family similarly affected.

The hair on the scalp was moderately thick, but showed a

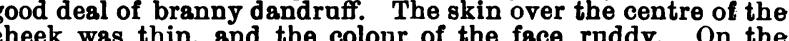
temples and surface underneath there was some dry seborrhoea. The skin from the neck to half-way down the thighs, and to within 2 in. of the wrist, was dry, wrinkled, and covered over with thick epidermic accumulations, presenting a dirty grey hue. He was fairly well nourished, yet the skin felt thin when pinched up, and there was no great amount of subcutaneous fat. The integument behind the knee, at the bend of the elbow, in the centre of each axilla, and in the fold of the groins was soft and entirely free from trace of ichthyosis. The shins were smooth but hairless, and the bands and feet exhibited no abnormality. Otherwise the child was healthy. The urine was normal.

Washed twice a day with the resorcin and salicylic soap and subsequently anointed with the same resorcin glycerine of starch as the previous cases, his condition on December 28th was as follows: The skin of the arms showed the rosy mottling of infantile health on exposure. Everywhere the skin was natural to feel, but on the abdomen, loins, and back was brownish in tint. On the flanks it showed fine, hair-like, atrophic lines, including oblong areas of normal skin. The thinning of the integument had largely disappeared, and he looked plump and much better nourished. There were no hairs visible anywhere on the body or limbs, and that on the scalp was still more scanty than is usual at his age. In January, 1907, Dr. Calvert was kind enough to ascertain his condition from his mother, who wrote as follows :

"The treatment has been carried out and so long as it is used the ichthyosis disappears. It is still used after his bath, but if omitted the roughness would return as before. $\mathrm{He}$ is fresher, plumper, stronger, and better in health at present than ever. There are fine hairs on the arms from the elbow to the wrist, and on the legs from the knee to the foot, but none on the rest of the body."

In the following instance the palms and soles were implicated with the rest of the cutaneous surface:

W. S, nearly 4 years. Admitted to my ward from the neighbourhood of Edinburgh on October 25th, 1904. His mother maintained that as a baby he had a healthy and fine skin, and that it was only after an attack of measles in January of that year that anything abnormal was observed. During the summier he had, in addition, whooping-cough, bronchitis, and jaundice, and, concurrently with these, the state of the skin got steadily worse. On admission the skin was universally dry, rough, and unperspiring. On the scalp there were thick, dirty yellowish, firmly-adherent flakes. There was intertrigo of the groins, accompanied with boils. The epidermis of the palms and soles was extremely hard, yellow, and dense, while cracks across the fingers limited their movements. In all other respects the child was bealthy.

To the groins were applied at first boric starch poultices for a few days, and then strips of zinc ichthyol salve muslin were interposed between the surfaces. This was persevered in till healing occurred, and subsequently these parts, as the general surface, were treated in the same manner as the previous case. On November 14 th the face was plump and quite smooth. The scalp was entirely devoid of crusts or scales, and was becoming closely covered with hair. With exception of the palms and soles, the integument of the body and limbs was perfectly soft and natural, while a fine lanugo hair had appeared on the arms and legs, absent on admission. The skin of the palms and soles was still drier than normal, and felt somewhat harsh to touch, but this did not interfere with free flexion or extension, and there were no cracks visible.

He was sent home shortly after, and the skin of the body and hands remained soft and pliant, requiring little attention, till his death from pneumonia a year later.

In the following instance regions not generally invaded by the ichthyotic - process were attacked, while there were also other peculiar features : 
M. L., 13, a native of Perthshire, was admitted to my ward october 27th, 1903. She was a well-grown girl, and had three brothers and one sister, but none of these nor any discoverable relative is or was similarly affected. According to her mother's account, the disease commenced when she was her mother's account, the disease commenced when she was These went away for a time and came back. Next the portion of the skin now implicated became dry and discoloured, especially the palms and soles.

The scalp was free from scales, the hair plentiful and auburn in hue. The face showed nothing abnormal. The palms exhibited elevated areas of enormously thickened epidermis, quite $\frac{1}{4}$ in. deep and sharply defined. Between these areas were spaces or fissures floored by integument apparently natural. The same condition extended about 1 in. up the front of the wrist, but the backs of the hands presented nothing unusual. At the bend of each elbow there was an oval area, 3 in. long by 2 in. broad, accurately marked off from the surrounding healthy skin. This area was black in tint, and had an aspect like very coarse plush, consisting of epidermic spicules closely set and standing up vertically. To the finger these gave the impression of a coarse, rough, shorthaired brush. Fine long hairs were interspersed between the spicules. The same condition was seen at the margins of the axillae and within the axillae, except in the very centre, where the skin was smooth over the space of an inch. The nipples and 'their areolae, the flanks, the front of the abdomen but avoiding the umbilicus, the groins but not the pubic region the patellar area, the popliteal space, the ankles and roots of the toes were covered with the same black, filiform, epidermic growth. The soles were overlapped with hard, dry, epidermic plates, more than $\frac{1}{4}$ in. thick, which broke away in places. Some of the nails were distorted and rough.

The treatment adopted for the body and limbs was the same as in the previous case, and need not be reiterated, but in order to afford every opportunity of thinning down the hypertrophied epidermis of the palms and soles, so far at least as to render it possible that when again at home there might be reasonable chance of its being kept in a fairly pliant condition she was retained in the ward till April 17th, 1904. To reduce the tylosis various methods were tried in succession. Thus the parts were enveloped in boric starch poultices for weeks, and attempts made to remove the soddened layers by scraping them with a sharp spoon, or by grinding them down with pumice stone. But no great progress was accomplished in this way. The epidermis, no doubt, was softened and rendered oedematous in this way, but at the same time it grew tender, while it did not become markedly thinner. Next, the continuous application of a resorcin plaster muslin was essayed but this failed to occasion exfoliation, and after a prolonged test was abandoned. On the whole, the application of $\epsilon$ mol keleet as a thick watery paste, under an impervious dressing of jaconet, proved the most successful of all. After six weeks of this combined with friction with pumice stone, the thickness of the epidermis was very considerably reduced, movement was free, while the troublesome fissures had grown much fewer. On the soles there was now only one distinct crack across the root of the toes, the rest of the surface was fairly soft, smooth, and pliant. The palms were, however, in hardly so satisfactory a condition, there were still many and wide fissures present. Some of these showed at their base an almost normal epithelial surface, others were narrow and painful, apt to bleed if stretched. Yet the hands could now be closed and opened after a fashion, which was quite impossible when she was admitted. For a short time thyroid tablets were given by Dr. Norman Walker during my absence. No perceptible effect was produced on the ichthyosis, while there was general discomplort and increased rapidity of the pulse. Its further employment was therefore given up.

Everywhere on the body the ichthyosis was reduced to a minimum. There was no trace remaining on the nipples or their areolae, where it had been so pronounced. The groins and the popliteal spaces presented a natural aspect. At the margins of the axillae, in the bend of the elbows and in front of the neck, there was nothing more than a slight yellowish, ridgy undulation. All the thready, papillary warty growths had disappeared, though the surface still had a pale yellow tint. The hairs seen at the bend of the elbow were still visible. The skin of the backs of the hands was now specially thin and transparent, so that the veins shone conspicuousl through. She had become much fatter, and the skin and scalp hair were healthy and well nourished. Though the application of resorcin had been continued throughout, there never was any sign of absorption, shown by darkening of the urine. When she was discharged, her parents were directed to wash her daily with resorcin and salicylic soap all over, hands and feet included, and to rub the palms and soles with pumice stone, after drying.

Whatever may be the hidden cause of ichthyosis, it is evident that it must be regarded as an exaggerated development of epidermis, or, as Thibierge well expresses it, "a hyperkeratosis with atrophy of the deep lavers of the rete mucosum." It is doubtful if the quasi-inflammatory appearances described by Unna and Tommasoli are not accidental rather than inherent characteristics. Its essence consists, then, in undue retention of the corneous layer, and not, as Brocq, usually so accurate, describes it, "as a disease characterized by incessant epidermic desquamation." 2 In fact exfoliation is in abeyance. As to the cause of this retention we are quite in the dark, antenatal pathology has so far failed to elucidate it, and the attractive epitrichium theory of Dumesnil and Bowen is negatived by the last case related, since in the very situations where according to it separation of the epitrichium occurs earliest, as in the axilla, and hence the ichthyosis should be absent, hyperkeratosis was especially noticeable.

In treatment, therefore, our efforts must be directed to promote and ensure regular systematic exfoliation of the unduly adherent and effete horny cells. There are various substances which have the power of thinning down, artificially, the epidermis. Sulphur is one, but if used it not only adds to the abnormal aridity, but is apt to set up exudative and even inflammatory processes. Salicylic acid, too, in the dilute form in which alone it can be prudently employed over extensive areas and for a length of time, fails to accomplish what is needed. Resorcin, however, not only favours continual desquamation but tends to leave the subjacent surface polished and pliant; hence its use is specially indicated. Combined with an oily base it dries up or is rubbed off. Glycerine from its hygroscopic properties is the excipient par excellence, but applied alone is rather irritating. In union with starch it forms a bland, persistent, soothing, and softening medium, and, as has been seen, while in all cases so used it proves eminently beneficial, if its application is begun early enough and steadily persevered in, it can effect what must be regarded as a cure.

At the same time, with all its qualifications, the resorcinized glycerine of starch alone would not enable us to get continuously rid of the ever newly-forming accretions of epidermis. We must therefore have recourse to a medicated soap, and a superfatted one with which resorcin and salicylic acid are incorporated-that which is so valuable in shortening the desquamative stage in scarlet fever - has proved adequate, and prepares the way for the subsequent glycerinization.

Internal remedies are of little use in ichthyosis. The only one which aids us at all is cod-liver oil administered in small doses at night. Pilocarpine is inoperative in early treatment, unnecessary in later.

$$
\begin{aligned}
& \text { REFERENCES. } \\
& 1 \text { La Pratique Dermatologique. Paris, 1901. Tome ii, p. 840. }{ }^{2} \text { Traité }
\end{aligned}
$$
Elementaire de Dermatologie Pratique, Paris, 1907, Tome ii, p. 686.

\section{THE TREATMENT OF RODENT ULCER BY} ZINC IONS.

\section{A Lecture delivered at the Medical Graddates'} Collegre and Polyclinic.

BY H. LEWIS JONES, M.D., F.R.C.P., MEDICAL OFFICER IN CHARGE OF THE ELECTRICAL DEPARTMENT,
ST. BARTHOLOMEW'S HOSPITAL.

I HAVE much pleasure in bringing forward the subject of the treatment of rodent ulcer by zinc ions, because it is not only new but good. Of late years the treatment of rodent ulcer has been robbed of many of its difficulties by the discovery of the effects of $x$ rays and of radium. Both of these agencies give good results when applied to rodent ulcer; in fact they cure the disease in a large proportion of cases, yet they have certain drawbacks. For instance, in the case of radium, the material is extremely costly; and in the case of the $x$ rays, an elaborate apparatus is required, and there is also a certain amount of risk attending both procedures, as their effect upon the tissues is difficult to control. The method which I am about to describe is a simple one; it can be carried out with an ordinary portable battery, and the results which it yields are quick and good.

The process is based upon the principle of the introduction of zinc, or rather of ions of zinc, into the tissues of the ulcer by means of a continuous current. The transportation of the zinc ions into the tissue of the affected part seems to exercise a profound influence upon rodent ulcer, causing it to assume the appearence of an ordinary simple sore, and, in many instances, to heal up in about a fortnight after a single application.

Before proceeding further, I shall ask you to look at a patient who had a rodent ulcer of the nose for five years. She gave the history that the sore had never healed since its com- 